\title{
Flexible Wearable Antenna on Electromagnetic Band Gap using PDMS substrate
}

\author{
Adel Y. I. Ashap ${ }^{1}$, Z. Z. Abidin ${ }^{\star^{2}}$, S.H. Dahlan ${ }^{3}$, H. A. Majid ${ }^{4}$, S.K. Yee ${ }^{5}$, Gameel Saleh $^{6}$, \\ Norun Abdul Malek \\ 1,2,3,4,5 Research Center of Applied Electromagnetics, Faculty of Electrical and Electronic Engineering, \\ Universiti Tun Hussein Onn Malaysia (UTHM), Batu Pahat, Johor, Malaysia \\ ${ }^{6}$ Biomedical Engineering Department, College of Engineering, University of Dammam, Dammam 1982, \\ Saudi Arabia \\ ${ }^{7}$ Kulliyah of Engineering, Electrical and Computer Engineering Department, International Islamic University \\ Malaysia (IIUM), P.O. Box 10, 50728 Kuala Lumpur, Malaysia \\ *Corresponding author, e-mail: zuhairia@uthm.edu.my
}

\begin{abstract}
A robust and low-profile electromagnetic band-gap (EBG) based on flexible wearable antenna covering $2.4 \mathrm{GHz}$ frequency band is presented. The incorporated EBG with antenna reduces the radiation into the human body around $17 \mathrm{~dB}$ and decreases the impacts of frequency detuning. The overall dimension of the antenna integrated with EBG is $56 \times 56 \times 4 \mathrm{~mm} 3$ with relative impedance bandwidth of $8.3 \%$ is achieved. The proposed design has improved the gain up to $7 \mathrm{dBi}$. Specific absorption rate (SAR) assessment is also studied to certify the performance of the antenna when it is located proximity to human tissue. The flexible antenna with aforementioned performances could be chosen as a good candidate for integration into a range of wearable devices for medical application.
\end{abstract}

Keywords: EBG, wearable antennas, PDMS, SAR

Copyright @ 2017 Universitas Ahmad Dahlan. All rights reserved.

\section{Introduction}

Wearable antennas are vital element for wireless devices in a body-area network (BAN). They obtain a numerous used by health care suppliers because of their potential relevance in different fields, such as tracking, smart houses, observing of human vital signs, patient, and battlefield survival [1]. As a rapidly growing research field, wearable antennas have grown an awesome amount of interest from both researchers; industry and academia due to their nontraditional operating environment-placed in extremely close proximity to the human body tissue. Accordingly, a demand of high radiation efficiency antenna while loading on human body makes the design a challenging task especially when it is required to be a low-profile, lightweight and robust characteristics. Furthermore, the influence of the wearable antenna on human body, characterized by the specific absorption rate, (SAR) [2-3] needs to take into account with safety level to insure that there is no impact on the human bodies and the value is minimized.

Over the previous decade, various antenna types have been investigated and documented for their suitability as wearable antennas including inverted-F antennas [4], cavitybacked or planar waveguide fed slots [5, 6], patch antennas [7] and monopole antennas [8]. Nevertheless, these antenna have disadvantages either/or high profile, narrow bandwidth and large footprints, that result in limited used in the application of wearable devices. More recently, electromagnetic band-gap (EBG) surfaces integrated with wearable antenna have been proposed and reported to enable and provide a high degree of isolation from the human body [1, 9-12]. However, some drawbacks still presents in the current EBG backed antenna designs such as poor front-to back ratio [11], relatively electrically large [9,10], rigid [12] and semi flexible [1].

In this paper, a compact flexible and robust wearable antenna incorporated with a compact footprint and low-profile EBG for wearable medical application is presented using polydimethylsiloxane (PDMS) as substrate. 


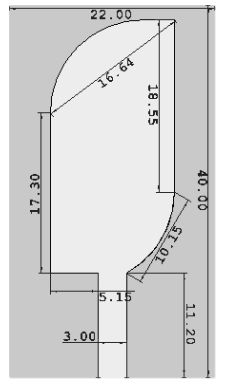

(a)

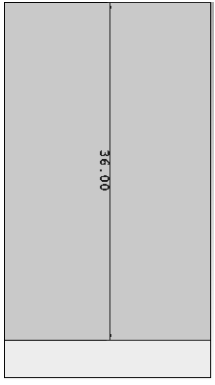

(b)

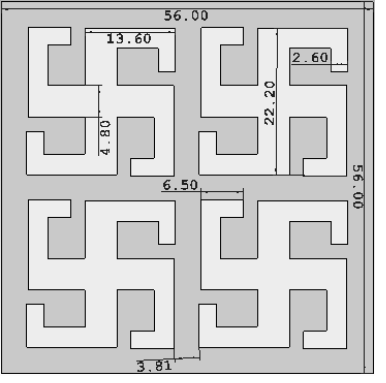

(c)

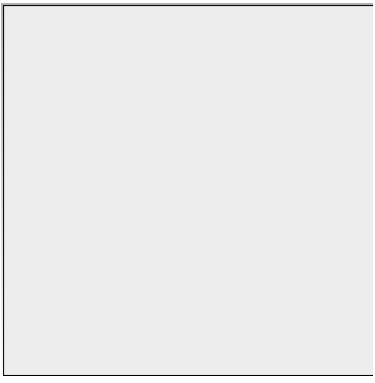

(d)

Figure 1. Geometry and dimensions (a) top view of monopole antenna, (b) bottom view of monopole antenna, (c) top view of the EBG structure, and (d) metallic backing view of the EBG structure

\section{Antenna and EBG Design}

The proposed microstrip monopole antenna is designed on $1 \mathrm{~mm}$ thick PDMS with a dielectric constant of 2.8 and loss tangent of 0.02 as showed in Figure 1 (a) and (b). The overall dimension of the antenna is $40 \times 22 \mathrm{~mm}^{2}$. A copper tape as conductive materials with thickness of $0.1 \mathrm{~mm}$ is used as metallic layer.

The antenna is placed on a top of EBGs array that works as an artificial magnetic conductor (AMC). The benefit of the used of $A M C$ is help to improve the performance of the antenna when it is located on the human body. The EBG array consists of $2 \times 2$ elements constructed on the same PDMS substrate as the antenna with $2 \mathrm{~mm}$ thick as depicted in Figure 1 (c) and (d). $1 \mathrm{~mm}$ Rohacell foam is inserted between antenna and EBG to avoid short circuit and to reduce the impedance mismatch. The overall dimension is $56 \times 56 \times 4 \mathrm{~mm}^{3}$.

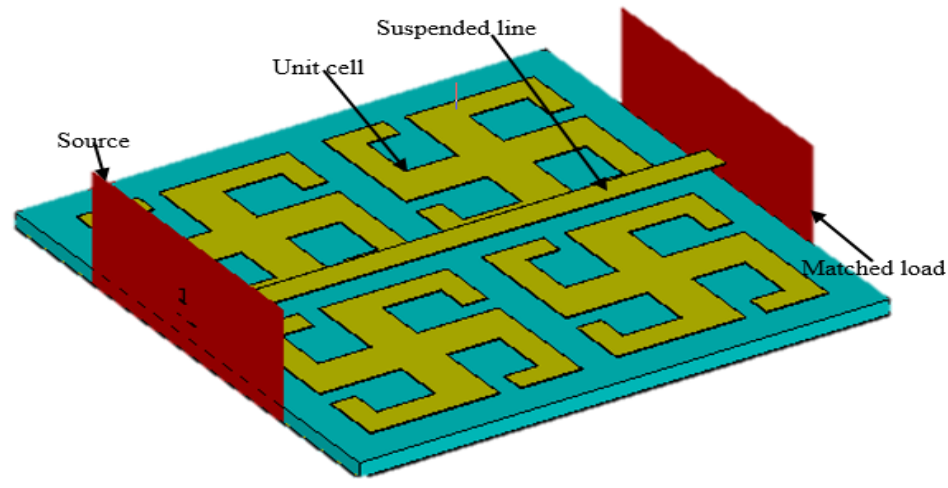

Figure 2. Suspended microstrip line method.

\section{Analysis and Discussion}

Two methods been used to investigate the EBGs characteristic at the demand frequency; (a) suspended transmission line and (b) reflection phase methods [13]. For the suspended transmission line method, an insulate microstrip transmission line is positioned over the EBG to be characterized as shown in Figure 2. Meanwhile, for the reflection phase technique, the boundaries of the BEG unit cell model are defined as unit cell as to represents an infinite structure. All the simulations process are performed in CST Microwave Studio ${ }^{8}$ [14]. From Figure 3 , it can be observed that the proposed EBG structures can reflect normally incident electromagnetic wave with a zero phase shift at a frequency of $2.4 \mathrm{GHz}$ (black color), while the suspended line examined a sufficiently well-defined band gap in the range of 2.36 $\mathrm{GHz}$ to $2.52 \mathrm{GHz}$ (red color). 


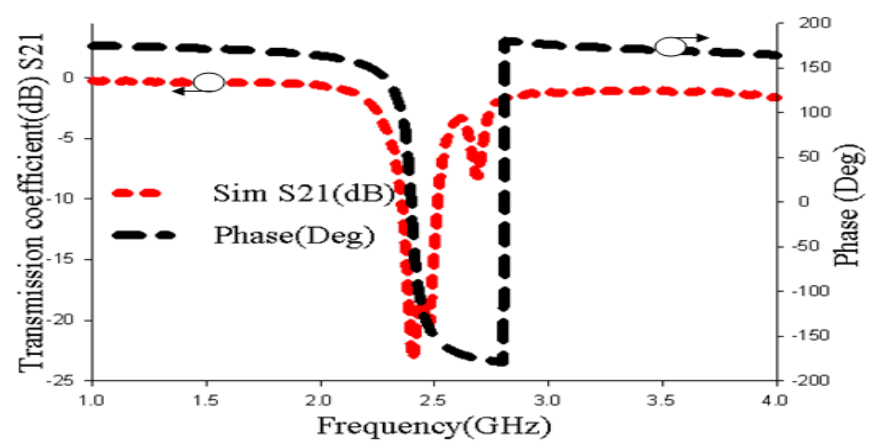

Figure 3. Simulated band gap response EBG using suspended line and reflection phase

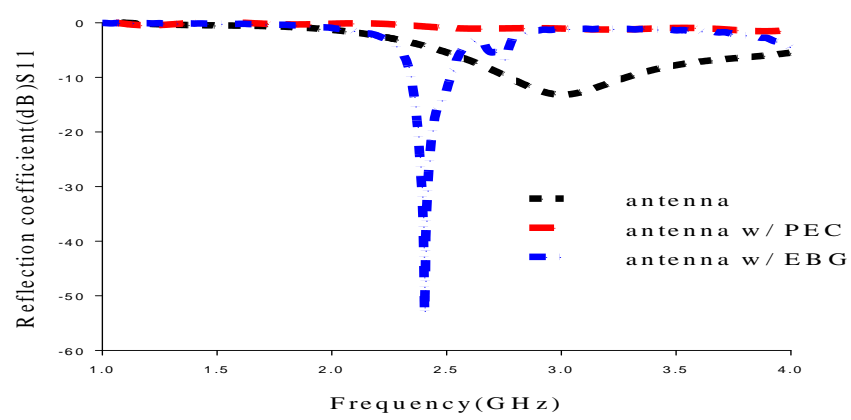

Figure 4. Reflection coefficient, $\mathrm{S}_{11}$ of antennas

The simulated results of the antenna alone and antenna with PEC and EBG in free space are illustrated in Figure 4. A poor impedance matching is observed when the antenna alone and with PEC as realized by the reflection coefficient, $\mathrm{S} 11$ higher than $-4.2 \mathrm{~dB}$ and -0.67 $\mathrm{dB}$ respectively. Integrating $\mathrm{EBG}$ to the antenna have its own benefit, in contrast, demonstrates reasonable impedance matching with reflection coefficient, $S_{11}$ lower than $-10 \mathrm{~dB}$ covering the spectrum bandwidth between $2.3-2.5 \mathrm{GHz}$ that specifies $8.3 \%$ relative bandwidth that sufficient to cover the MBANs band. From Figure 5 (a), it can be seen that the simulated gain of the antenna alone and antenna with PEC is approximately $2.09 \mathrm{dBi}$ and $6 \mathrm{dBi}$ respectively, compared to the antenna with EBG with gain of $7 \mathrm{dBi}$ was achieved. Furthermore, the analysis of the front to back ratio (FBR) for the three cases are studied as showed in Figure 5(b). It can be observed that in the case of antenna alone and antenna with PEC the FBR are close to zero and $5.9 \mathrm{~dB}$ respectively, whereas by integrating EBG enabled antenna achieves the FBR of 18 $\mathrm{dB}$.

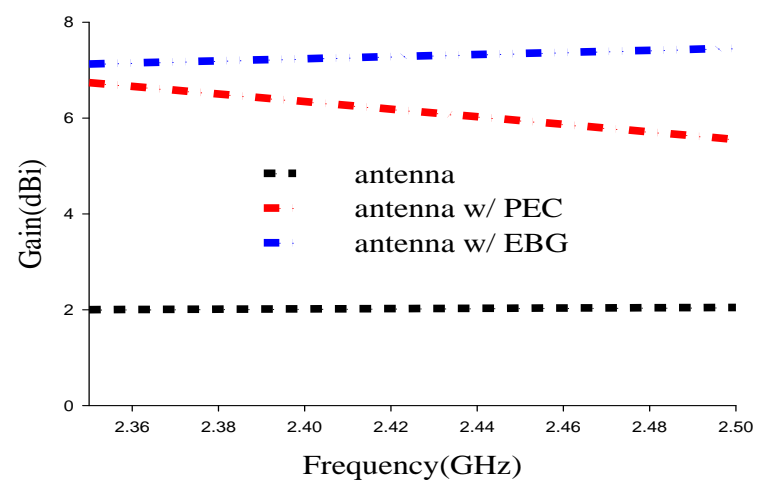

(a)

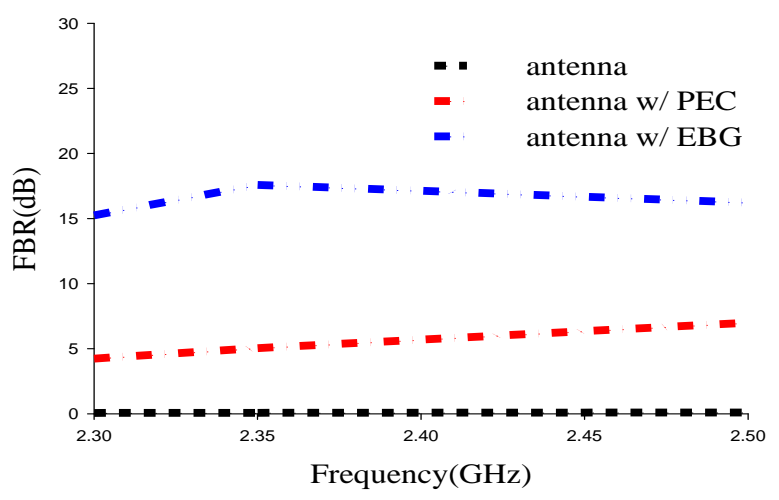

(b)

Figure 5. Simulated monopole antenna (a) gain, and (b) front back ratio (FBR) 
The analysis of the radiation pattern at $\mathrm{E}$-plane and $\mathrm{H}$-plane are demonstrated when the antenna alone and antenna with PEC and EBG in free space as depicted in Figure 6 . It is noticed that the antenna with PEC has almost broadside radiation pattern even the gain was acceptable as depicted in Figure 5(a). This is due to destructive that cause by the image current of PEC that has an opposite direction to the original current of the antenna resulting in cancelation each other. Additionally it also effect the reflection coefficient, $\mathrm{S}_{11}$ by having value greater than $-10 \mathrm{~dB}$. While the radiation patterns are realized decreased towards the body when the EBG is added to the antenna, furthermore back lobe reduction at $2.4 \mathrm{GHz}$ is $18 \mathrm{~dB}$ compare when the antenna alone.

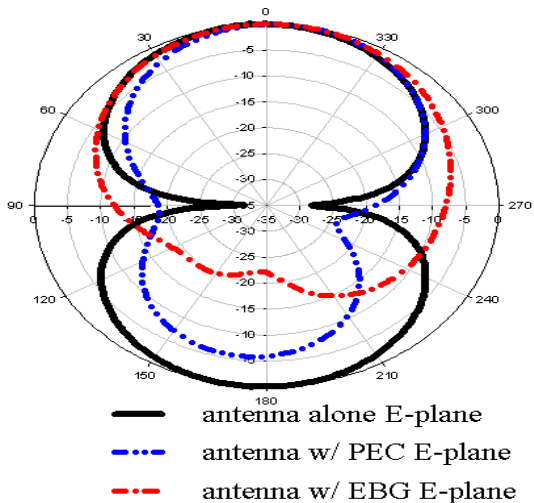

(a)

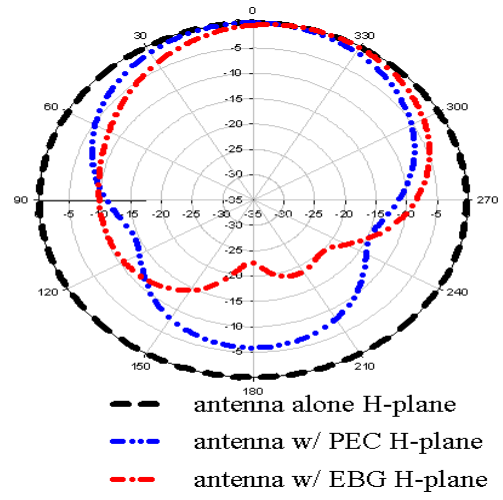

(b)

Figure 6. Simulated radiation pattern characteristics

\section{Performance of the Prroposed Desgn on Body}

Since the introduced antenna is designed for wearable applications, it is vital to describe the performance of the antenna EBG on-body in points of interest. Consequently, the subsequent section assessing the antenna under bending conditions is initially followed by SAR evaluation.

\subsection{Bending Evaluation}

As to satisfy that wearable antenna is conformal to human body surfaces, an examination of antenna bending for various level of structure is required before an investigation of human body loading impacts. The influence of bending is assessed by simulating the antenna over cylindrical model with different diameters, $R$ in the $y$-axes as illustrated in Figure 7. The results of S11 are shown in Figure 8. It is seen that as the diameter decreases, the result of $S_{11}$ is increasing especially when $R=70 \mathrm{~mm}$ due to the direction of bending, which is not parallel to the current on the patch. Nevertheless, the demand band still achieve by showing the $S_{11}$ is less than $-10 \mathrm{~dB}$. The existent of ripples are expected due to the effect of impedance matching during the bending.

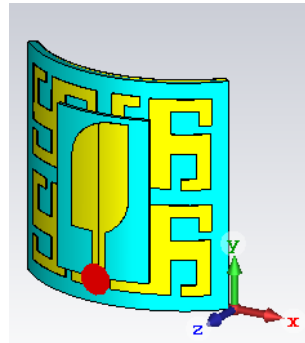

$\mathrm{R}=70 \mathrm{~mm}$

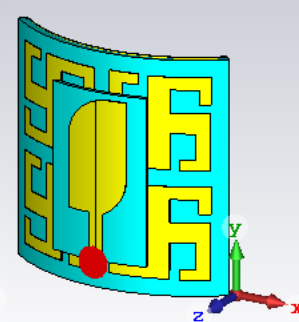

$\mathrm{R}=80 \mathrm{~mm}$

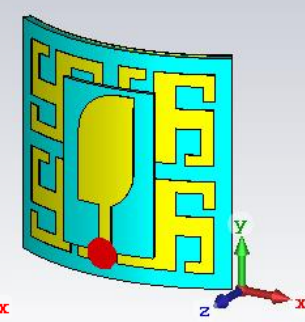

$\mathrm{R}=100 \mathrm{~mm}$

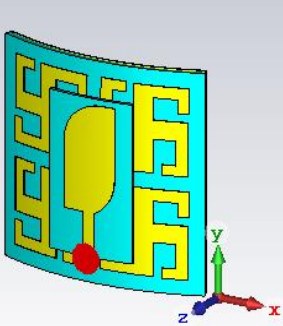

$\mathrm{R}=140 \mathrm{~mm}$

Figure 7. Structurally bending integrated EBG-enabled antenna with different diameters 


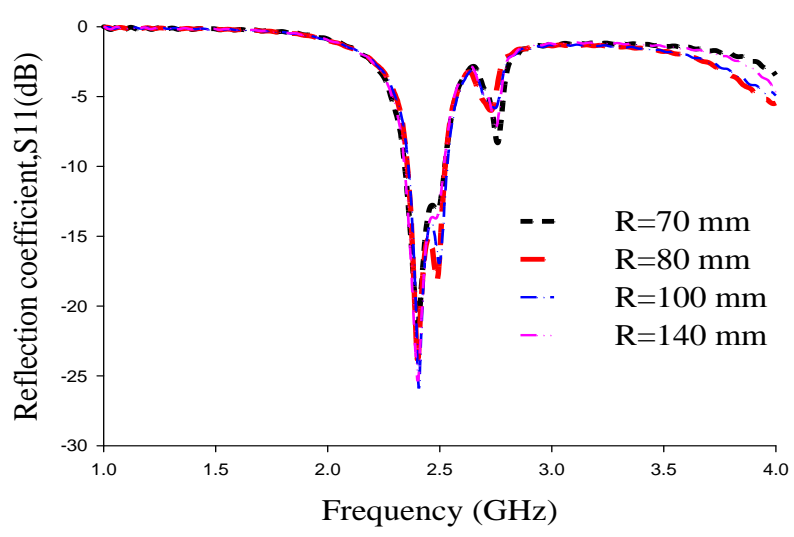

Figure 8. Reflection coefficient,$S_{11}$ of integrated EBG-enabled antenna on bending effects with difference diameters

\subsection{Specific Absorption Rate (SAR)}

SAR investigation was carried out using CST MWS simulator. This has been demonstrated by located a human model in the vicinity of the incorporated antenna with EBGs. The cylindrical model was used as to mimic the human arm with multilayer human tissue. The model has an outer radius of $40 \mathrm{~mm}$ and consist of a $13 \mathrm{~mm}$ thick bone layer with relative permittivity of 18.49 and conductivity values of $0.82 \mathrm{~S} / \mathrm{m}$, a $20-\mathrm{mm}$-thick muscle layer with relative permittivity of 52.67 and conductivity values of $1.77 \mathrm{~S} / \mathrm{m}$ a $5-\mathrm{mm}$-thick fat layer with relative permittivity of 5.27 and conductivity values of $0.11 \mathrm{~S} / \mathrm{m}$, and 2-mm-thick skin layer with relative permittivity of 37.95 and conductivity values of $1.49 \mathrm{~S} / \mathrm{m}$. This data has been taken from previously reported studies of [14]. The model is placed $1 \mathrm{~mm}$ from the incorporated antenna with EBG ground.

The input power used to analyze the SAR value is $100 \mathrm{~mm}$ as a benchmark. The calculation is based on the IEEE C95.1 standard and averaged over $1 \mathrm{~g}$ of biological tissue. Based on the guidelines specified by FCC standard, the SAR values must be not exceeding 1.6 $\mathrm{W} / \mathrm{kg}$ averaged over $1 \mathrm{~g}$ of tissue [15].

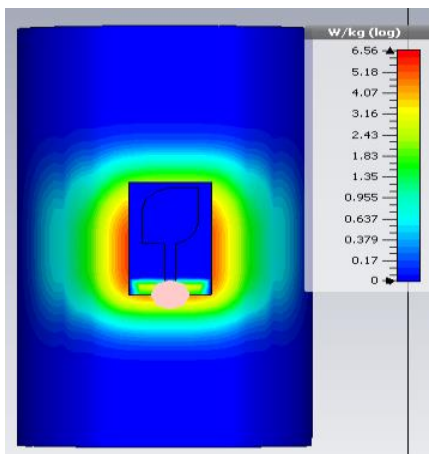

(a)

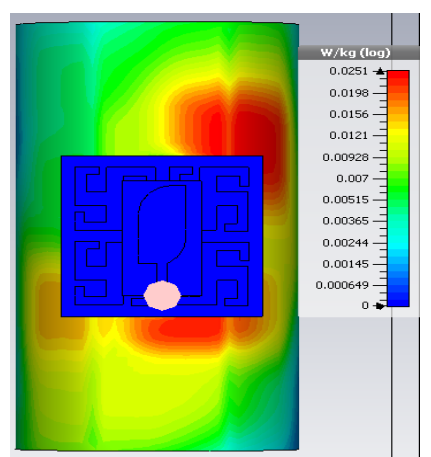

(b)

Figure 9. Simulated SAR values for (a) the antenna alone and (b) the integrated EBG-enabled antenna at $1 \mathrm{~mm}$ away from the tissue model

The SAR performance is compared between the antenna alone and with EBG as shown in Figure 9. The antenna has maximum $1 \mathrm{~g}$ averaged SAR value of about $6.6 \mathrm{~W} / \mathrm{kg}$ because of its omnidirectional radiation characteristic. Even the antenna is placed at $5 \mathrm{~mm}$ away from the model tissue but still realized a maximum $1 \mathrm{~g}$ averaged SAR value as high as $5.3 \mathrm{~W} / \mathrm{kg}$,, as seen 
in Table 1. Adding EBG to the antenna, a more reduction is noticed in the maximum $1 \mathrm{~g}$ averaged SAR value dropping to $0.03 \mathrm{~W} / \mathrm{kg}$ even when the antenna with EBG is only $1 \mathrm{~mm}$ away from the model tissue. Furthermore, EBG has benefited the antenna by showing a significant reduction of SAR that has been achieved. Table 2 shows the comparison of various SAR values obtained with and without the EBG structure presented by previous researchers.

Table 1. Maximum $1 \mathrm{~g}$ averaged SAR value for the antenna, the integrated EBG with antenna at different distances away from the tissue model (units: $\mathrm{w} / \mathrm{kg}$ )

\begin{tabular}{cccc}
\hline Distance & $1 \mathrm{~mm}$ & $3 \mathrm{~mm}$ & $5 \mathrm{~mm}$ \\
\hline Antenna & 6.6 & 6 & 5.3 \\
Antenna with EBG & 0.03 & 0.02 & 0.01 \\
\hline
\end{tabular}

The influence on the antenna performance at several distances from the human tissue model for antenna a lone and with EBG are shown in Figure 10 (a) and Figure 10(b) respectively. It is obvious that the reflection coefficient, $S_{11}$ for the incorporated antenna with EBG shows reasonable $S 11$ results compared to the antenna alone. It can be noticed that, from Figure 10(a), the resonance frequencies are shifted proportionally by the distance, $d$. This is might be due to the conductivity of body with the antenna. Additionally, the gain of the incorporated structural is well-maintained when it is located on the human model, showing a stable gain of $6.8 \mathrm{dBi}$.

Table 2. Shows the various SAR values obtained with and without the EBG structure

\begin{tabular}{ccc}
\hline Ref & SAR Value of antenna $(\mathrm{w} / \mathrm{kg})$ & SAR Value with EBG $(\mathrm{w} / \mathrm{kg})$ \\
\hline$[12]$ & 13.5 & 0.29 \\
{$[1]$} & 16.8 & 0.66 \\
{$[11]$} & 1.88 & 0.683 \\
{$[9]$} & 13.85 & 0.079 \\
This work & 6.6 & 0.03 \\
\hline
\end{tabular}

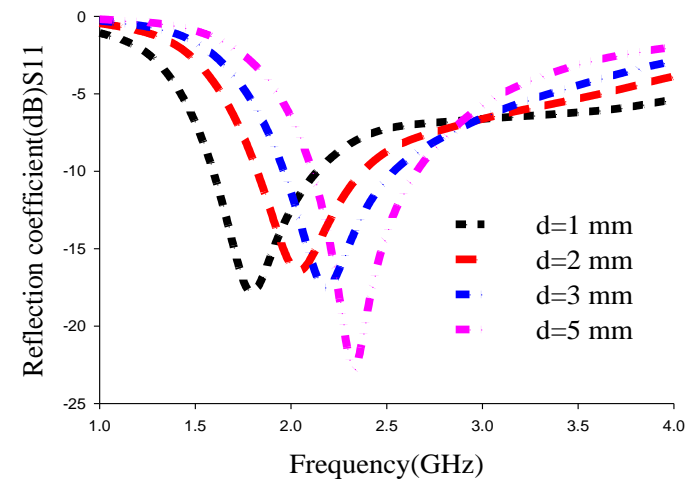

(a)

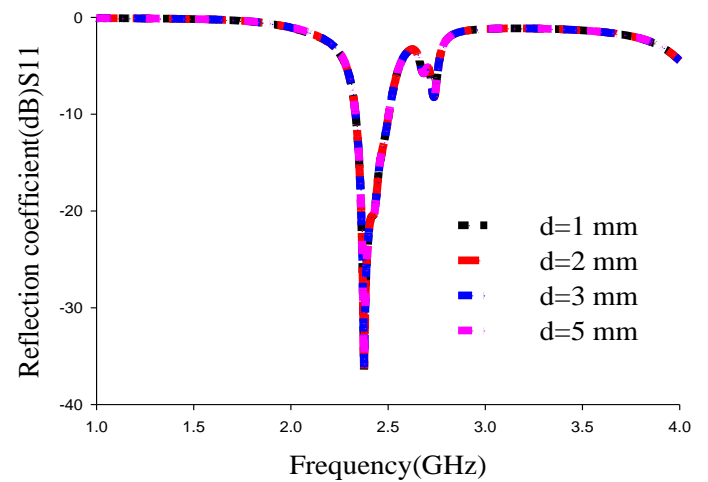

(b)

Figure 10. Simulated $S_{11}$ for (a) the antenna alone and (b) the integrated EBG-enabled antenna at different distances (d) away from the tissue model

\section{Conclusions}

A conformal flexible incorporated antenna with EBG with a low-profile, robust and compact footprint, has been introduced for wearable applications. It was clearly that the presented EBG integrated antenna achieved an impedance bandwidth of $8.3 \%$ with $7 \mathrm{dBi}$ gain. The impacts under bending condition of the design were demonstrated. The integrated design has shown a good performance when it was loaded to human body by maintaining a good return loss and reduced SAR values. 


\section{Acknowledgement}

The authors of this paper wish to acknowledge the funding of this project by Ministry of Education Malaysia (MOE) under Research Accularation Collaborative Effort (RACE) Vot No. 1510 and GPPS Vot No. U739.

\section{References}

[1] Z Jiang, DE. Brocker, PE. Sieber, DH Werner. A compact, low-profile metasurface-enabled antenna for wearable medical body-area network devices. IEEE Trans. Antennas Propag. 2014; 62(8):40214030.

[2] RA. Abd-Alhameed, PS. Excell, MA. Mangoud. Computation of specific absorption rate in the human body due to base-station antennas using a hybrid formulation. IEEE Trans. Electromagn. Compat. 2005; 47(2):374-381.

[3] RA. Abd-Alhameed, PS. Excell, AM. Mangoud. A Hybrid Computational Electromagnetics Formulation for Simulation of Antennas Coupled to Lossy Dielectric Volumes. IEEE Trans. Broad. 2004; 50(3):253-259.

[4] PJ. Soh, GAE. Vandenbosch, SL. Ooi, NHM. Rais. Design of a broadband, all-textile slotted PIFA. IEEE Trans. Antennas Propag. 2012; 60(1): 379-384.

[5] N Haga, K Saito, M Takahashi, K Ito.Characteristics of cavity slot antenna for body-area networks. IEEE Trans. Antennas Propag. 2009; 57(4):837-843.

[6] R Moro, S Agneessens, H Rogier, M Bozzi. Wearable textile antenna in substrate integrated waveguide technology. Electron. Lett. 2012; 48(16):985.

[7] A Alomainy, Y Hao, A Owadally, CG Parnini, Y. Nechayev, CC Constantinou, PS. Hall. Statistical analysis and performance evaluation for on-body radio propagation with microstrip patch antennas. IEEE Trans. Antennas Propag. 2007; 55(1): 245-248.

[8] PS. Hall, Y. Hao, YI. Nechayev, A. Alomainy, CC. Constantinou, C. Parini, MR. Kamarudin, TZ. Salim, DTM. Hee, R. Dubrovka, AS. Owadall, W. Song, AA. Serra, P. Nepa, M. Gallo, M. Bozzetti. Antennas and propagation for on-body communication systems. IEEE Antennas Propag. Mag.vol. 2007; 49(3) 41-58.

[9] S. Zhu, R. Langley. Dual-band wearable textile antenna on an EBG substrate. IEEE Trans. Antennas Propag .2009; 57(4): 926-935.

[10] S. Velan, EF. Sundarsingh, M. Kanagasabai, AK. Sarma, C. Raviteja, R. Sivasamy, JK. Pakkathillam. Dual-Band EBG Integrated Monopole Antenna Deploying Fractal Geometry for Wearable Applications. IEEE Ant. Wire. Propag. Lett. 2015; 14: 249-252.

[11] HR. Raad, Al. Abbosh, HM. Al-Rizzo, DG. Rucker. Flexible and compact AMC based antenna for telemedicine applications. IEEE Trans. Antennas Propag. 2013; 61(2): 524-53.

[12] YS. Chen, TY. Ku. A Low-Profile Wearable Antenna Using a Miniature High Impedance Surface for Smartwatch Applications, in IEEE Antennas and Wireless Propagation Letters. 2016; 15: 1144- 1147.

[13] Aminian A, F Yang, Y Rahmat-Samii, In-phase reflection and EM wave suppression characteristics of electromagnetic band gap ground planes, Proceedings of the IEEE Antennas and Propagation Society International Symposium. 2003; 4:430-433.

[14] CST Microwave Studio [Online]. Available: http/www.cst.com.

[15] MA. Stuchly, SS. Stuchly .Dielectric properties of biological substances-tabulated. J. Microw. Power.1980; 15(1):19-26.

[16] http://www.fcc.gov/encyclopedia/specific-absorption-rate-sar-cellulartelephones 